\title{
Supply Chain Finanzierung in der Automobilindustrie
}

\section{Wie OEMs mithilfe unternehmensübergreifender Working Capital- Praktiken die Wettbewerbsfähigkeit ihrer Wertschöpfungsnetzwerke zukünftig verbessern können}

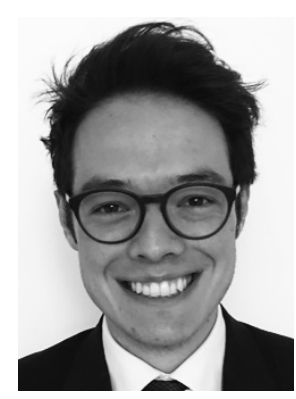

Philipp Wetzel, M.A. HSG, ist Projektmanager sowie PhD Candidate am Institut für Supply Chain Management der Universität St. Gallen. E-Mail: philipp.wetzel@unisg.ch

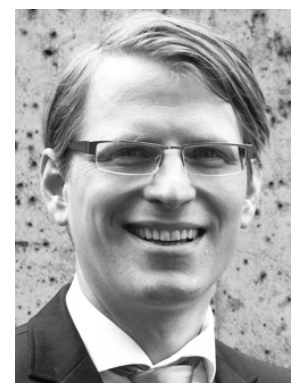

Prof. Dr. Erik Hofmann ist Direktor am Institut für Supply Chain Management der Universität St. Gallen. E-Mail: erik. hofmann@unisg.ch

\begin{abstract}
Unternehmensübergreifendes Working Capital Management (WCM) eröffnet Unternehmen vielfältige Möglichkeiten, um ihre Ziele im WCM gemeinsam mit Lieferanten und Kunden zu erreichen. Mithilfe von digitalisierten Supply Chain Finance-Lösungen können Unternehmen nicht nur ihre eigenen Bilanzkennzahlen, Abwicklungsprozesse und Finanzierungskosten nachhaltig verbessern, sondern auch die Wettbewerbsfähigkeit ihrer Supply Chain Partner stärken. Anhand des Beispiels der Automobilindustrie wird in diesem Beitrag verdeutlicht, wie ein unternehmensübergreifendes WCM unter Einbezug von Supply Chain Finance zum Erfolgsfaktor für die gesamte Supply Chain avanciert.
\end{abstract}

\section{Philipp Wetzel, Erik Hofmann und Felix Köpple}

\section{Unternehmensübergreifendes WCM mit Supply Chain Finance}

In der Praxis wird mit Blick auf die Optimierung des Working Capitals in der Breite oftmals eine einseitige Verbesserungsstrategie verfolgt: „Verbindlichkeiten so spät wie möglich bezahlen, Forderungen so schnell wie möglich eintreiben und gleichzeitig Bestände senken“. Die negativen Auswirkungen auf vor- und nachgelagerte Supply Chain-Partner, die eine solche einseitige Optimierung des Working Capitals mit sich bringt, werden dabei häufig unterschätzt. Die Resultate einer aktuellen wissenschaftlichen Studie zum Working Capital Management (WCM) deuten darauf hin, dass zwischen der Unternehmensperformance und dem Niveau an Nettoumlaufvermögen (Working Capital) nicht ein negativ linearer, sondern ein invertierter U-förmiger Zusammenhang besteht (vgl. Wetzel/ Hofmann, 2019). Die allgemeine Lehrbuchmeinung im WCM „less is always more“ scheint damit überholt. Insbesondere in der Automobilindustrie zeigt sich, dass eine Reduktion des Nettoumlaufvermögens nur bis zu einem gewissen Niveau einen positiven Effekt auf die Unternehmensperformance ausübt. Stellt man beispielsweise das Working Capital-Level (gemessen mit dem C2C-Cycle in Tagen) der Profitabilität (gemessen in ROCE in Prozent) von Tier 1 Lieferanten der größten OEMs einander gegenüber, so wird deutlich, dass die Lieferanten in der „Mitte“ ihr Working Capital am „optimalsten“ einsetzen.

Konkret sind also jene Unternehmen, die sich auf der linken Seite des Working Capital-Optimums befinden (vgl. Abb. 1, Punkt 1) dazu angehalten, ihr Working Capital-Niveau z. B. zugunsten ihrer Lieferanten zu vergrößern. Analog sollten Unternehmen, die sich auf der rechten Seite des Optimums befinden (vgl. Abb. 1, Punkt 2) eine (kooperative) Verringerung des Working Capitals anstreben. Das adäquate Niveau an Working Capital ist für die Unternehmen somit erreicht, wenn die positiven und negativen Effekte der Kapitalbindung im WCM unter Einbezug der Lieferanten- und Kundenseite bestmöglich ausbalanciert sind. Wichtig dabei ist zu berücksichtigen, dass das unternehmensspezifische optimale Niveau an Nettoumlaufvermögen von verschiedenen Treibern und Einflussfaktoren abhängt.

Doch mit welchen Praktiken erreichen Unternehmen ihr performancemaximierendes Niveau an Working Capital bei gleichzeitiger Berücksichtigung der Interessen von Kunden und Lieferanten? Die kurze Antwortet lautet: Supply Chain Finance (SCF). 


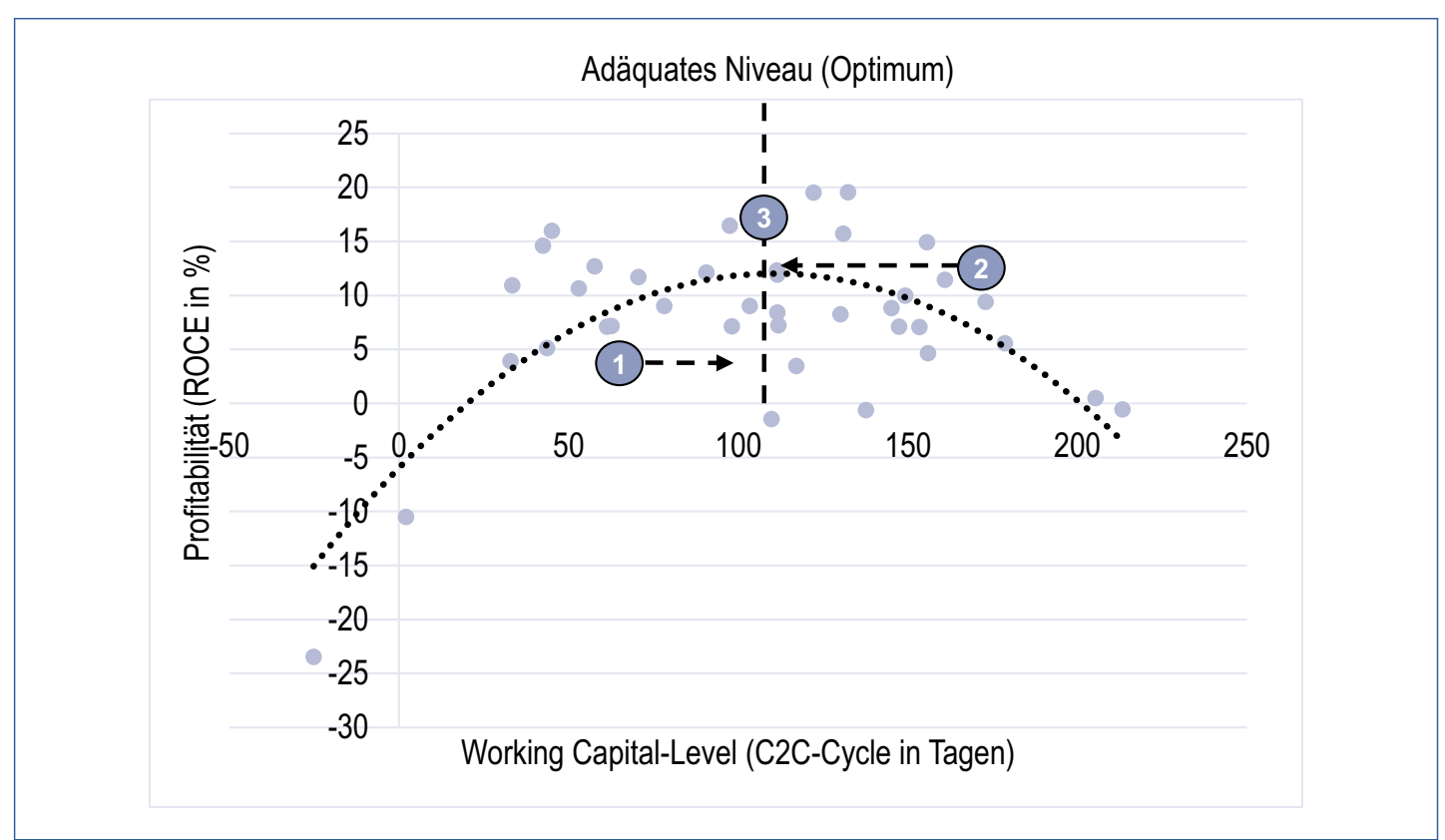

Abb. 1: Zusammenhang zwischen Working Capital-Level und Profitabilität von Tier 1 Lieferanten der Automobilindustrie (Datenbank: Orbis, 2017; insgesamt 39 Tier 1 Lieferanten)

SCF kann als Sammelbegriff für verschiedene Working Capital-Finanzierungslösungen verstanden werden, welche die unternehmensübergreifende Optimierung des Nettoumlaufvermögens sowie die Integration der Finanzprozesse zwischen Lieferanten, Kunden und externen Dienstleistern (z. B. Banken, Fintechs oder Logistikdienstleistern) fokussiert (vgl. Hofmann/Wetzel, 2018). Abb. 2 zeigt, dass derzeit eine Reihe von innovativen SCF-Lösungen auf dem Markt existieren, die sich verschiedenster digitaler Möglichkeiten bedienen, um diese Integrationsleistung zu erfüllen. SCF hat damit den Anspruch, die unterschiedlichen Interessen und finanziellen Zielsetzungen entlang des Wertschöpfungsprozesses auszugleichen und die Bilanz und Erfolgsrechnung für alle beteiligten Supply ChainAkteure zu verbessern (vgl. Wetzel/Movcharenko, 2019). Eine Schlüsselrolle kommt der Digitalisierung der WCM-Prozesse zu, da diese die Basis für Supply Chain-weite WCM-Optimierungskonzepte über die eigenen Unternehmensgrenzen hinweg darstellt. So können einkaufende Unternehmen (Abnehmer) mit ausreichendem Liquiditätsüberschuss mithilfe einer SCF-Plattform beispielsweise ihre vorteilhafte Finanzierungssituation dazu nutzen, Lieferanten mit beschränkten liquiden Mitteln bei der Finanzierung ihres Working Capitals zu unterstützen.

\section{Infobox: Kurzbeschreibung ausgewählter SCF- Lösungen \\ Kreditorenbereich}

- Procurement-Cards (P-Cards) stellen eine Form der Firmenkundenkarte dar (häufig im Kreditkarten-Format) und ermöglichen es, Waren und Dienstleistungen ohne einen traditionellen Einkaufsprozess bei vordefinierten Lieferanten zu be-

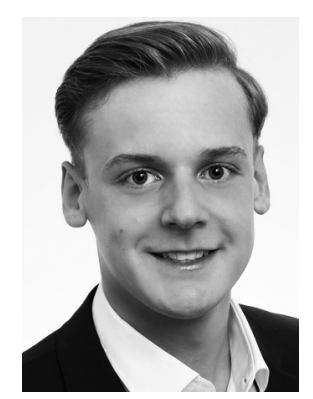

Felix Köpple, B.A. HSG, ist studentischer Mitarbeiter am Institut für Supply Chain Management der Universität St. Gallen.

schaffen. P-Cards werden in der Regel für kleinere Einkäufe verwendet, um eine erhöhte Kosteneffizienz und Kontrolle zu erreichen.

- Purchase-Order Financing (Finanzierung von Bestellungen) beschreibt eine SCF-Finanzierungslösung, die zwischen Käuferunternehmen und Lieferanten vereinbart werden kann, um eine frühzeitige Finanzierung (zum Zeitpunkt der Bestellung) des Lieferanten zu erreichen.

- Dynamic Discounting (dynamische Diskontierung) beschreibt eine SCF-Lösung, bei der ein Käuferunternehmen dem Lieferanten eine frühere Zahlung der Rechnung anbietet. Im Gegensatz zum herkömmlichen Skonto beinhaltet Dynamic Discounting dynamische Zahlungsmodalitäten und wird in der Regel über eine am Markt verfügbare SCF-Plattform abgewickelt.

- Reverse Factoring stellt eine klassische SCF-Lösung dar, die im Wesentlichen eine Vorfinanzierung der Verbindlichkeiten eines Abnehmers ge-

Zentrale Aussagen

- Einseitige und „unkooperative" Working Capital-Initiativen, wie beispielsweise die systematische Verlängerung von Zahlungszielen gegenüber Lieferanten, gilt es generell zu vermeiden, da diese auf Kosten der vorgelagerten Supply Chain-Partner erzielt werden und in Form des "Working Capital Bumerang-Effektes” auf das eigene Unternehmen zurückfallen.

- Wie das Beispiel aus der Automobilindustrie zeigt, senkt das unternehmensübergreifende Working Capital Management die Finanzierungs- und Herstellungskosten im Wertschöpfungsnetzwerk und erhöht dadurch die Wettbewerbsfähigkeit von allen beteiligten Unternehmen nachhaltig.

- Supply Chain Finance stellt ein flexibles Finanzierungsinstrument dar, das Unternehmen gezielt zur Anvisierung des performancemaximierenden Niveaus an Net Working Capital einsetzen können. 


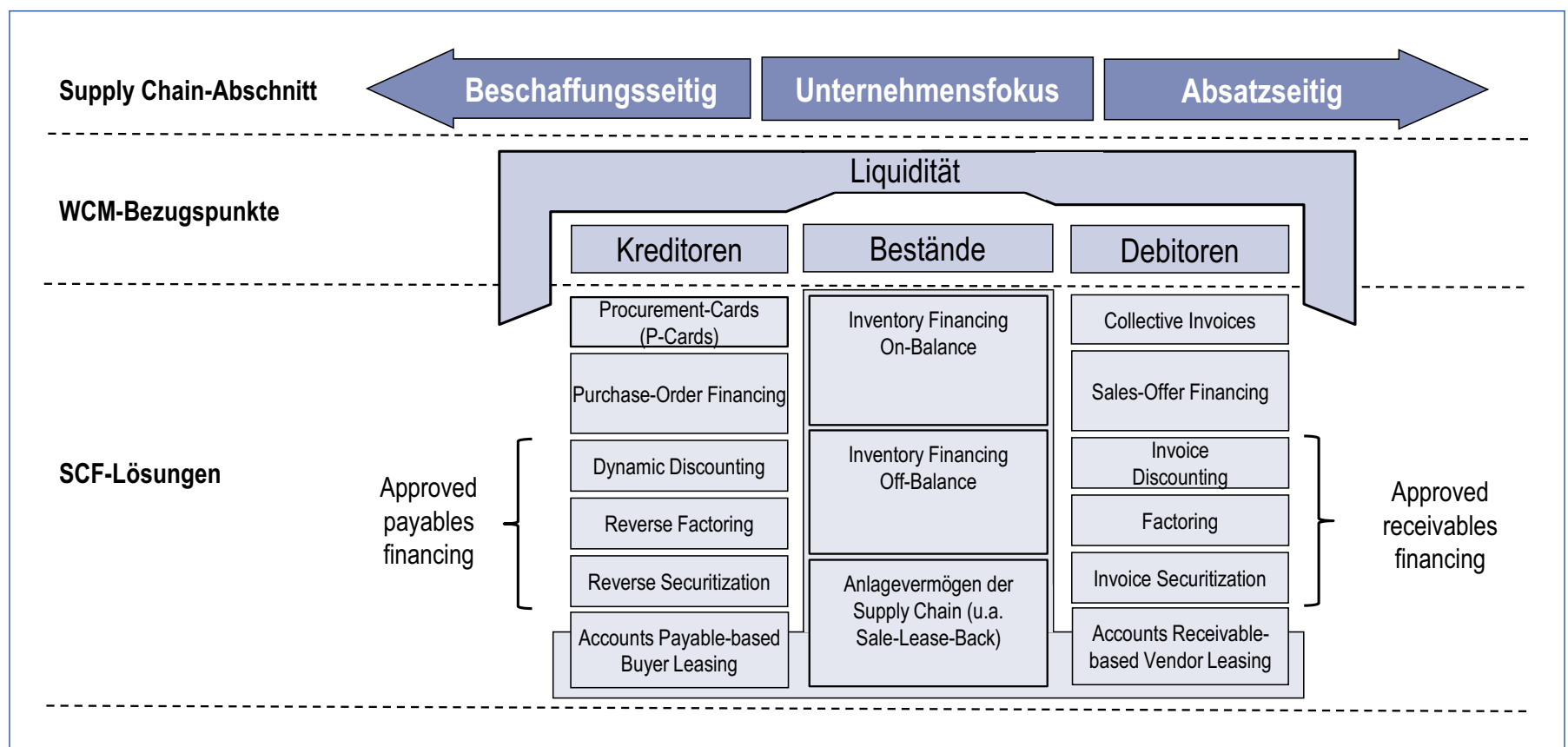

Abb. 2: Ausgewählte SCF-Lösungen für fokale Unternehmen

genüber seinem Lieferanten ermöglicht. Beim Reverse Factoring schließt ein Factoring-Anbieter einen Vertrag mit einem Abnehmer von Waren (Käuferunternehmen), in dem er sich dazu verpflichtet, die Forderungen von dessen Lieferanten vorzufinanzieren.

Mit den auf dem Markt verfügbaren SCF-Lösungen lassen sich inzwischen alle WCM-Bereiche optimieren.

- Reverse Securitization stellt eine SCF-Lösung dar, bei der sich im Gegensatz zur klassischen Forderungsverbriefung (Invoice Securitization) das gesamte Kreditrisiko auf das initiierende Käuferunternehmen konzentriert. Die zu finanzierenden Vermögenswerte werden dabei mit einem Abschlag an eine Zweckgesellschaft (SPV) verkauft und im Anschluss an private und institutionelle Anleger am Kapitalmarkt veräußert.

- Accounts Payable-based Buyer Leasing (Beschaffungsleasing) beschreibt eine Finanzierungslösung zur Reduktion der investitionsbedingten Kapitalbindung von Käuferunternehmen. Beschaffungsleasing ermöglicht dadurch eine nutzungsorientierte Bereitstellung von Investitionsgütern.

\section{Bestandsbereich}

- Off-Balance Inventory Financing (bilanzwirksame Bestandsfinanzierung) steht für die Finanzierung von Beständen, wobei die operative Warenlogistik und das Eigentum an einen externen Logistikdienstleister übertragen werden. Daraus resultieren eine Kostensenkung durch geringere Lager- und Logistikkosten sowie eine Liquiditätssteigerung durch eine Reduktion des in den Beständen gebundenen Kapitals.

- On-Balance Inventory Financing (bilanzunwirksame Bestandsfinanzierung) beschreibt ein Instrument zur Finanzierung von Beständen. Anders als bei der Off-Balance-Lösung bleiben bei der
On-Balance-Lösung die Bestände jedoch in der Bilanz des Käuferunternehmens und dienen lediglich als Sicherheit für einen Kreditvertrag (Asset-basedLending-Ansatz).

- Sale-Lease-Back beschreibt ein Verfahren, bei dem ein Unternehmen bewegliches oder unbewegliches Anlagevermögen der Supply Chain an einen Leasinggeber verkauft, um dieses im Anschluss wieder zurück zu leasen. Diese Sonderform des Leasings ermöglicht es Unternehmen, kurzfristig Liquidität freizusetzen.

\section{Debitorenbereich}

- Collective Invoices (Sammelgutschriften) ermöglichen es Unternehmen, für mehrere Bestellungen oder Aufträge von Kunden eine gemeinsame Rechnung zu erstellen bzw. Aufträge in einer Gutschrift zusammenzufassen.

- Sales-Offer Financing (Absatzfinanzierung) umfasst Finanzierungslösungen, die Lieferanten dazu nutzen, den Absatz ihrer Produkte zu forcieren und finanzielle Spielräume für Kunden $\mathrm{zu}$ schaffen (z. B. durch den Einsatz von Ratenzahlungen).

- Invoice Discounting (Rechnungsdiskontierung): Im Gegensatz zum klassischen Factoring werden beim Invoice Discounting die Debitorenrechnungen nicht an eine Factoring-Gesellschaft verkauft, sondern dienen lediglich als Sicherheit für eine kurzfristige Finanzierung (Asset-based-LendingAnsatz).

- Factoring beschreibt die gewerbliche, revolvierende Übertragung von Forderungen eines Unternehmens an einen Finanzdienstleister oder eine Factoring-Gesellschaft. Factoring als SCF-Lösung bietet Unternehmen eine Liquiditätssteigerung bei 


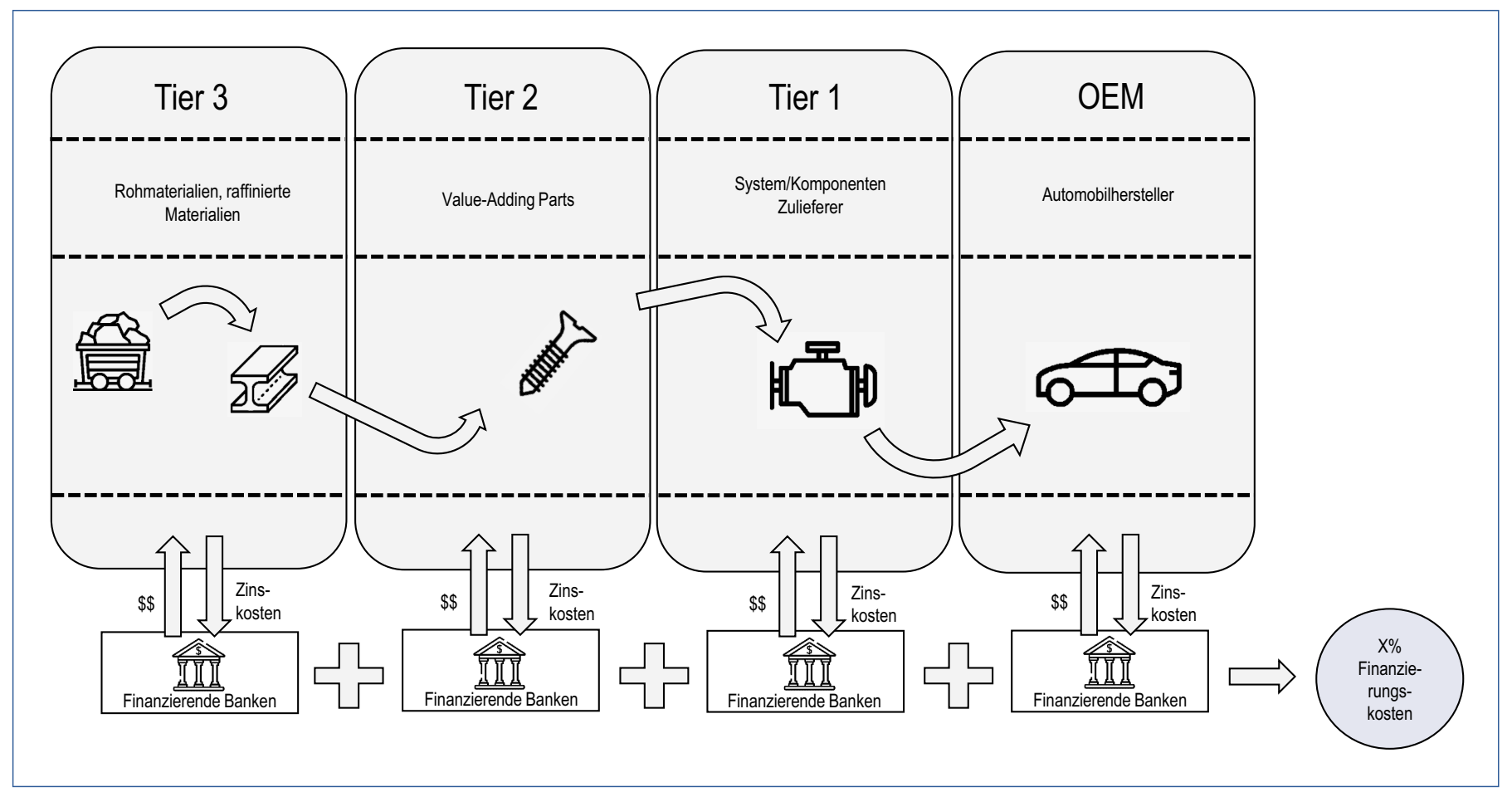

Abb. 3: Wertschöpfungsstufen-bezogene Finanzierungskosten entlang einer schematischen Automotive Supply Chain

gleichzeitiger Reduktion der Finanzierungskosten und Forderungsausfälle.

- Invoice Securitization (Forderungsverbriefung) beschreibt eine SCF-Lösung, bei der Forderungen verkauft werden. Im Gegensatz zum klassischen Factoring werden die Forderungen jedoch gebündelt und in kurzfristige Wertpapiere umgewandelt und mittels einer Zweckgesellschaft (SPV) einer breiten Investorenbasis auf dem Kapitalmarkt angeboten.

- Accounts Receivable-based Vendor Leasing (Absatzleasing) beschreibt eine Finanzierungslösung, die insbesondere beim Verkauf von Investitionsgütern eingesetzt wird. Absatzleasing ermöglicht es Unternehmen, kapitalintensive Produkte in Kombination mit einer Finanzierungslösung in einem Lösungspaket anbieten zu können.

\section{Status-quo zum WCM in der Automobil- industrie}

Angesichts der zunehmend international vernetzten Produktion im Zuge der Globalisierung ist das WCM in der kapitalintensiven Automobilbranche mit ihrer über 100-jährigen Geschichte ein Dauerthema. Stellten einst die Original Equipment Manufacturer (OEM) einen Großteil der zu verbauenden Teile selbst her, so führen die steigende Produktkomplexität in Verbindung mit sinkenden Margen zu einem umgreifenden Strategiewechsel weg von vertikaler Integration hin zu einem hohen Grad an Spezialisierung. So stellt ein OEM heute lediglich 30 \% des Endproduktes selbst her, wobei der Rest von vorgelagerten Supply Chain-Partnern bezogen wird (vgl. VDA, 2019). Daraus resultieren entsprechend komplexe, die ganze Welt umspannende Wertschöpfungsnetzwerke. Aus dem WCMBlickwinkel stellt dabei insbesondere die Optimierung der unternehmensübergreifenden Finanzprozesse hinsichtlich der Kapitalkosten zwischen Lieferanten und Kunden eine große Herausforderung dar. Abb. 3 illustriert schematisch eine beispielhafte Automotive Supply Chain, wobei ersichtlich wird, dass auf jeder Wertschöpfungsstufe etwaige Finanzierungskosten anfallen. Diese setzen sich aus verschiedensten Bestandteilen zusammen, wobei unter anderem die Opportunitätskosten des Working Capitals mit einfließen.

Wie die idealisierten Wertschöpfungsstufen andeuten, sehen sich die Tier 1 bis Tier 3 Lieferanten in der Automobilindustrie im Hinblick auf das WCM häufig mit dem Problem konfrontiert, dass OEMs ihre Marktmacht mitunter dazu ausnutzen, um einseitige Working Capital-Verbesserungen zu realisieren (z. B. mittels Verlängerung der Zahlungsziele). Diese Besonderheit spiegelt sich in den WCM-Kennzahlen der vier größten OEMs sowie der acht größten Automobilzulieferer in Deutschland wider. Wie in Abb. 4 ersichtlich, bestehen zwischen den zwei Gruppen von Unternehmen im Hinblick auf die Kapitalbindungsdauer - gemessen in Form des C2C-Cycle und seinen Komponenten markante Unterschiede. Während das Working Capital bei den Zulieferern durchschnittlich 63 Tage gebunden ist, beträgt die Kapitalbindung bei den OEMs durchschnittlich nur 47 Tage. Besonders auffällig ist dabei auch, dass die Zulieferer mit einem DSO von 56 Tagen im Durchschnitt mehr als doppelt so lange auf die Bezahlung ausstehender
Die Optimierung der unternehmensübergreifenden Finanzflüsse stellt eine große Herausforderung dar. 


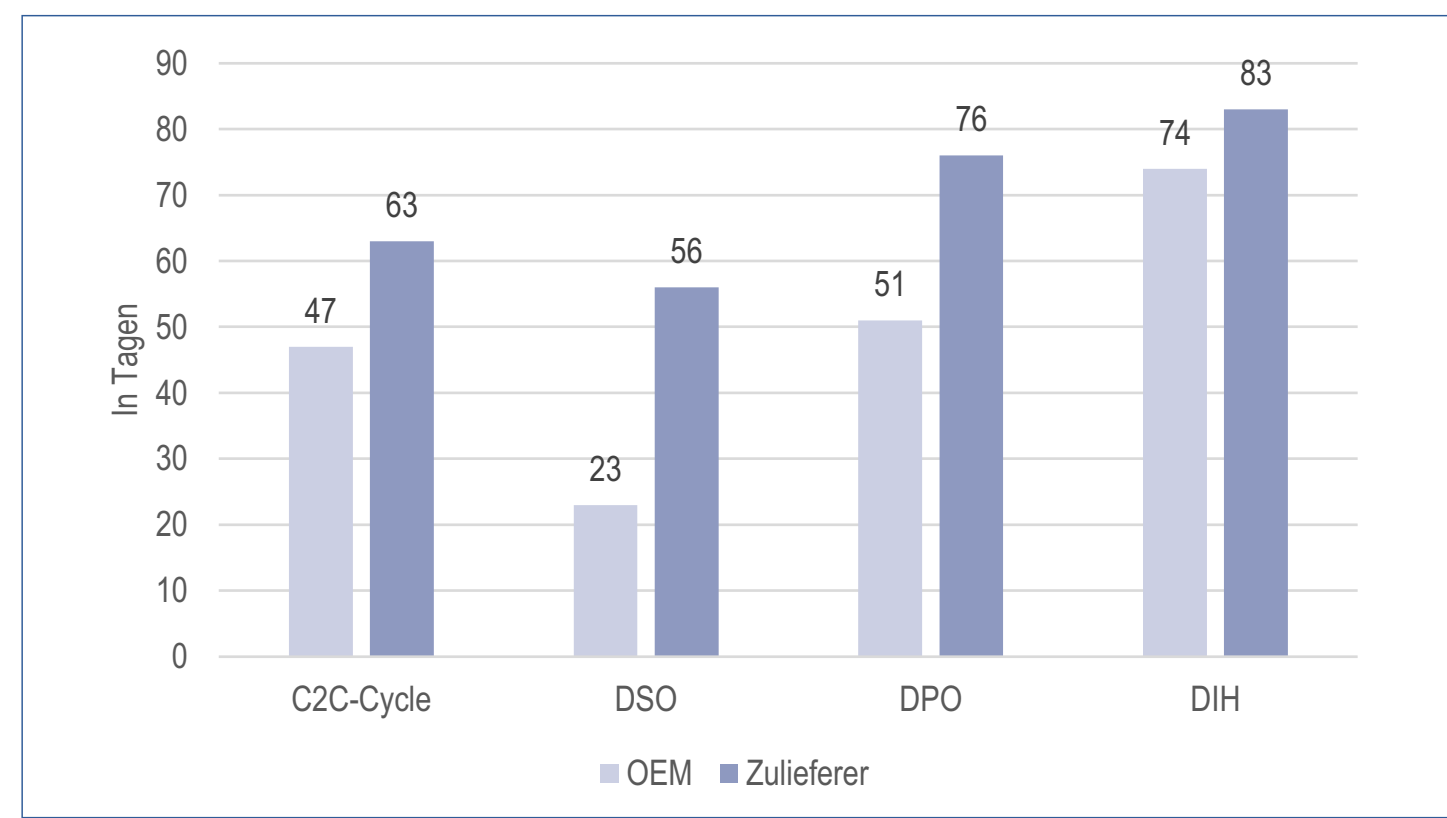

Abb. 4: WCM-Kennzahlenwerte deutscher Automobilunternehmen (Datenbank: Orbis, 2017; Durchschnittswerte der 4 größten OEMs und 8 größten Tier 1 Lieferanten)

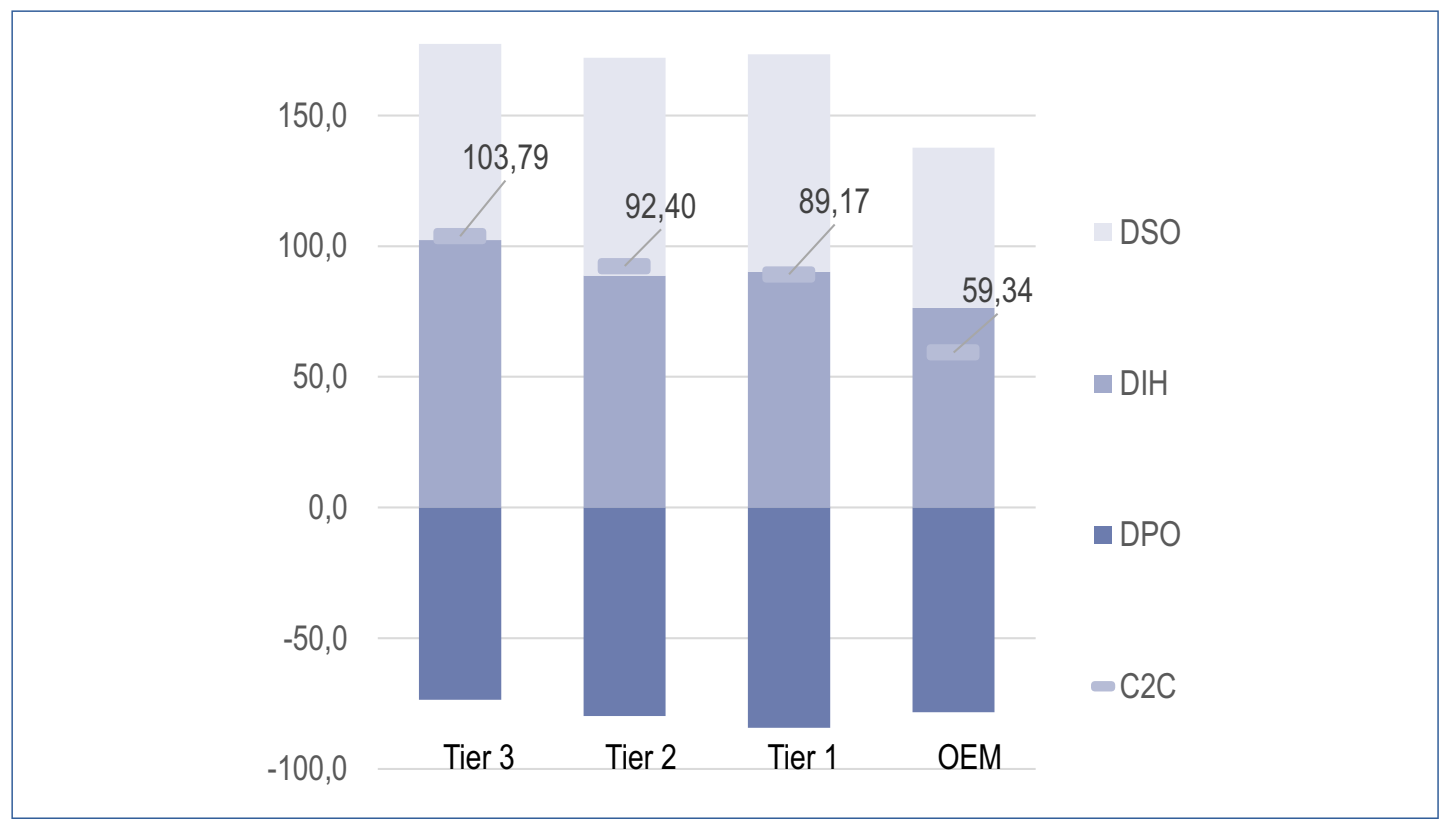

Abb. 5: WCM-Kennzahlenwerte der globalen Automobilindustrie (Datenbank: Orbis, 2017; Insgesamt 117 OEMS, 564 Tier 1 Lieferanten, 1565 Tier 2 Lieferanten, 1898 Tier 3 Lieferanten)

Hinsichtlich der zu tragenden Working Capital-Finanzierungslast bestehen entlang der Supply Chain markante Unterschiede.
Forderungen warten als die OEMs, die einen durchschnittlichen DSO von 23 Tagen aufweisen.

Doch nicht nur deutsche Automobilunternehmen versuchen ausstehende Rechnungen zulasten ihrer vorgelagerten Supply Chain-Partner möglichst spät zu bezahlen, um ihren eigenen C2C-Cycle zu verkürzen. Auch bei einem globalen Vergleich der WCM-Kennzahlenwerte von in der Automobilindustrie tätigen Unternehmen fallen deutliche Unterschiede entlang der Supply Chain ins Auge (vgl. Abb. 5). So zeigt sich, dass Tier 1 Lieferanten ihre Rechnungen im Durchschnitt noch später begleichen und somit Vorlieferanten, welche weiter in der Supply Chain zurückliegen, damit noch län- ger auf die Begleichung von Forderungen warten lassen. Sie schieben gewissermaßen die ihnen widerfahrenden Zahlungsziele auf ihre Lieferanten weiter. Allgemein lässt sich konstatieren, dass vorgelagerte Supply Chain-Partner mit zunehmender Entfernung zum OEM im Durchschnitt auch eine höhere Working Capital-Finanzierungslast zu bewältigen haben. Die durchschnittliche Kapitalbindungsdauer (gemessen anhand des C2C-Cycle) steigt entlang der Supply Chain kontinuierlich an: von 59 Tagen bei den OEMs bis zu 104 Tagen bei den Tier 3 Lieferanten. Dieser Umstand in Verbindung mit der Tatsache, dass weiter flussaufwärts in der Supply Chain liegende Unternehmen tendenzi- 


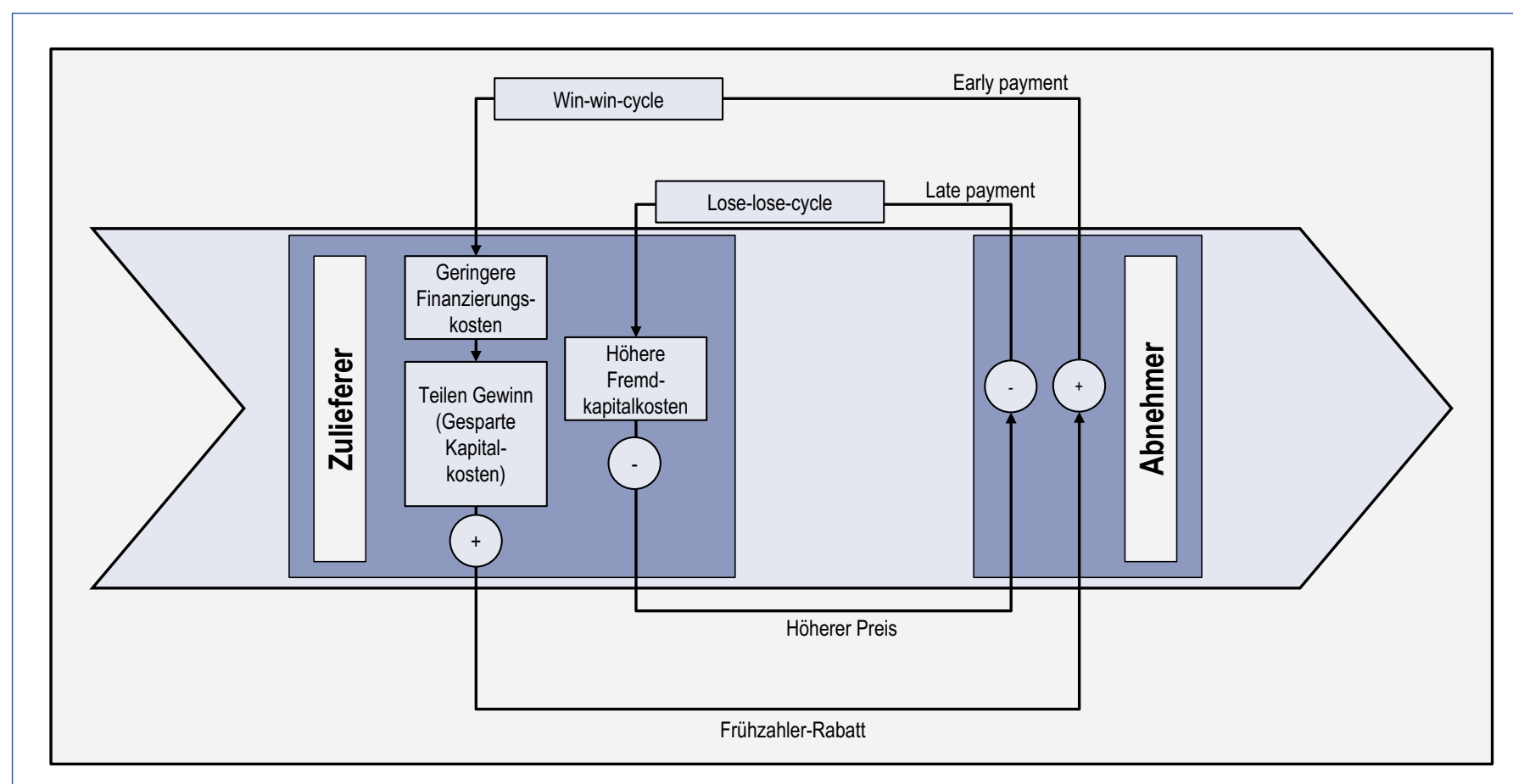

Abb. 6: Positive und negative Working Capital-Bumerang-Effekte in der Supply Chain

ell kleinere Akteure sind (somit tendenziell auch schlechtere Finanzierungskonditionen besitzen) führt aus einer übergeordneten Perspektive insgesamt zu einer ineffektiven Situation, welche sich durch einseitige Working Capital-Verbesserungen zulasten von vorgelagerten Supply Chain-Partner noch weiter verschlechtert.

\section{Working Capital-Bumerang-Effekte entlang der Supply Chain}

Aus Sicht einer vereinfachten Gesamtkostenkalkulation heraus, müssen Zulieferunternehmen anfallende Kapitalkosten in den Verkaufspreis ihrer Produkte einpreisen. Aus langfristiger Betrachtung bezahlt der OEM den Preis für ein (zu) spätes Begleichen der Rechnung (siehe Abb. 6: „Lose-lose-cycle“). Die vermeintlich eingesparten Finanzierungskosten besitzen somit Eigenschaften eines Bumeranges. Letzterer distanziert sich zunächst (die Rechnung wird spät beglichen, der vermeintlich „günstige“ Lieferantenkredit wird voll genutzt). Zu einem späteren Zeitpunkt kommt dieser jedoch in Form eines höheren Produktpreises wieder zurück. Da sich der Effekt mit den skizzierten Nachteilen von Stufe zu Stufe im Wertschöpfungsnetzwerk fortsetzt, ist von einer reduzierten finanziellen Stabilität in der gesamten Supply Chain auszugehen (vgl. Vazquez et al., 2016).

Der negative Working Capital-Bumerang-Effekt lässt sich jedoch durch eine Zusammenarbeit zwischen den Partnern der Supply Chain unterbrechen und eindämmen. Der Abnehmer mit seiner besseren Liquiditäts- und Finanzierungssituation begleicht ausstehende Rechnungen wesentlich früher, was das Working Capital der Zulieferer (welche i. d. R. einen tendenziell höheren Finanzierungssatz aufweisen) verringert, und somit gleichzeitig hilft, deren Finanzierungskosten zu senken. Die positiven Effekte beim Lieferanten lassen sich ihrerseits wiederum im Rahmen der Gewährung eines Skontos an den Abnehmer zurückgeben, was seitens des Abnehmers gleichzeitig zu einem reduzierten Finanzaufwand führt. Somit ergibt sich durch die schnellere Bezahlung ein sogenannter „Win-win-cycle“, welcher durch die Nutzung einer Supply Chain Finance-Lösung initiiert wird. Hinzu kommt, dass vor allem in Zeiten der gegenwärtigen Niedrigzinspolitik ein vorhandener Liquiditätsüberschuss in die eigene vorgelagerte Supply Chain reinvestiert werden kann und das zu verhältnismäßig attraktiven Renditeerwartungen.

Der Working Capital-Bumerang-Effekt lässt sich nicht nur zwischen den OEMs und deren direkten Tier 1 Zulieferern beobachten, er existiert auch zwischen den Tier 1 Lieferanten und deren Tier 2 Zulieferern (Vorlieferanten) usw. und reicht bis hin zum Anfang des Wertschöpfungsnetzwerks zurück (Rohstofflieferanten). Die Etablierung positiver Working Capital-Bumerang-Effekte entlang mehrerer Wertschöpfungsstufen wird auch als Deep-tier Supply Chain Financing bezeichnet.

\section{Zukunftsfähiges WCM mit Supply Chain Finance in der Automobilbranche}

Fortschrittliche OEMs sind sich der Working Capital-Bumerang-Effekte bewusst und haben erkannt, dass es langfristig nicht zielführend ist, die Kosten für Working Capital-Finanzierungen auf Supply
Negative Working Capital-BumerangEffekte lassen sich mithilfe von Supply Chain FinanceLösungen unterbrechen und eindämmen. 


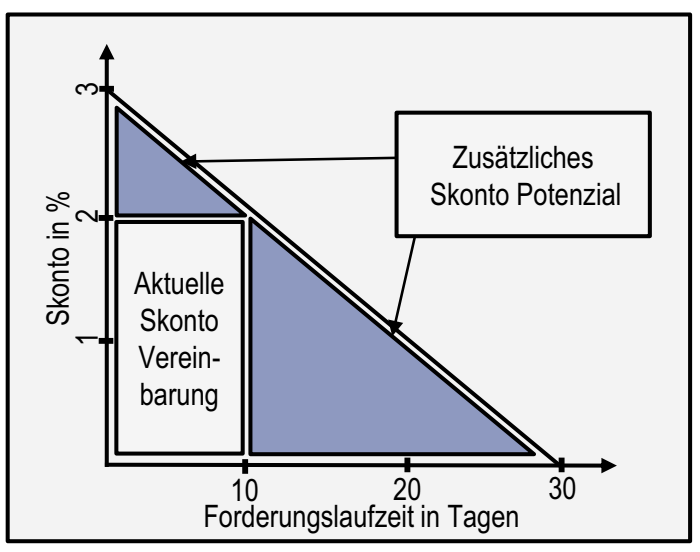

Abb. 7: Die möglichen Effekte des Dynamic Discountings

Die traditionelle Skonto-Praxis in Zulieferer-Abnehmer-Beziehungen lässt sich durch Dynamic Discounting deutlich erweitern.
Chain-Partner (insb. Zulieferer) abzuwälzen, da diese mittel- bis langfristig wieder auf das eigene Unternehmen zurückfallen können (z. B. auch in Form von Lieferausfällen). Unternehmensübergreifende Ansätze wie SCF sind in der kapitalintensiven Automobilindustrie deshalb im Trend, da sie die unterschiedlichen Working Capital-Bedarfe der Lieferanten und Vorlieferanten berücksichtigen und eine Steigerung der Wettbewerbsfähigkeit der gesamten Supply Chain ermöglichen.

Als klassisches Beispiel gilt die SCF-Lösung Dynamic Discounting, bei der OEMs ihren Lieferanten flexibel eine frühere Zahlung der Rechnungen anbieten können. Im Gegensatz zum herkömmlichen Skonto, in dem Lieferanten ihren Kunden pauschal $2 \%$ Skonto bei Bezahlung innerhalb von 10 Tagen anbieten (Trade Credit), ermöglicht Dynamic Discounting dynamische Zahlungsmodalitäten innerhalb einer Zulieferer-Abnehmer-Beziehung. Die traditionelle Skonto-Praxis lässt sich durch Dynamic Discounting deutlich erweitern, da durch An- wendung dieser SCF-Praktik auch beispielsweise $1 \%$ Skonto bei Zahlung innerhalb von 20 Tagen möglich ist (vgl. Abb. 7). Das Prinzip ist einfach: Je früher eine Rechnung bezahlt wird, desto höher fällt der „Abschlag“ für den Lieferanten aus.

Dynamic Discounting führt folglich zu einer deutlichen Erhöhung der Flexibilität des Trade Credit Einsatzes. Zudem beeinflusst die Nutzung des Dynamic Discountings die Dauer der Finanzierung bzw. die Länge des C2C-Cycles für den Lieferanten wie auch für den Abnehmer. Des Weiteren wird die Unsicherheit bezüglich des Working Capitals reduziert, indem es den Zulieferern ein präziseres Management der eingehenden Zahlungsströme ermöglicht. Aus der Sicht des Abnehmers kann Dynamic Discounting zudem gleichzeitig die beste Kapitalrendite für seine überschüssige Liquidität bieten. Dies ist insbesondere in der Automobilindustrie von großer Relevanz, da OEMs im Vergleich zu ihren Lieferanten im Normalfall deutlich tiefere durchschnittliche Kapitalfinanzierungskosten aufweisen (vgl. Abb. 8).

Moderne Dynamic Discounting-Lösungen werden in der Regel über eine am Markt verfügbare SCF-Plattform abgewickelt, wobei Letztere das Angebot sowie die Nachfrage nach der Skonto-getriebenen Liquidität miteinander verbinden (siehe z. B. Taulia (taulia.com), Tradeshift (tradeshift.com) oder CRX Markets (crxmarkets.com)). Vor allem die Zunahme an digitalen Rechnungsprozessen hat den Vorstoß solcher digitalen SCF-Lösungen ermöglicht. Die ERP-Systeme der beteiligten Supply Chain-Parteien werden über die Plattformen via standardisierter Schnittstellen miteinander nahtlos verbunden, wodurch sich die effektive Praktikabilität für alle teilnehmenden Akteure rasch herstellen lässt. So haben bereits erste OEMs

\begin{tabular}{|c|c|c|c|}
\hline & Unternehmen & WACC & Delta zu Daimler \\
\hline 离 & Daimler & $3.4753 \%$ & \\
\hline \multirow{10}{*}{ 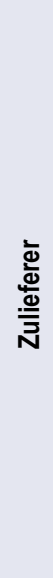 } & Magna International Inc & $9.9530 \%$ & $6.4777 \%$ \\
\hline & Continental AG & $8.9743 \%$ & $5.4990 \%$ \\
\hline & China Motor Corp & $8.4000 \%$ & $4.9247 \%$ \\
\hline & ZF Friedrichshafen AG & $6.1000 \%$ & $2.6247 \%$ \\
\hline & Lear Corp & $8.8214 \%$ & $5.3461 \%$ \\
\hline & Kuka AG & $11.6094 \%$ & $8.1341 \%$ \\
\hline & Cummins Inc & $10.5561 \%$ & $7.0808 \%$ \\
\hline & Arcelor Mittal & $8.8000 \%$ & $5.3247 \%$ \\
\hline & Cie Generale (Michelin) & $7.7000 \%$ & $4.2247 \%$ \\
\hline & Alphabet Inc & $12.1170 \%$ & $8.6417 \%$ \\
\hline
\end{tabular}

Abb. 8: Gewichtete durchschnittliche Kapitalkosten von Daimler und ausgewählten Zulieferern (2018) 
der Automobilindustrie mit der Einführung sogenannter „Early Payment“-Plattformen begonnen (z. B. C2FO.com), wobei sich Zulieferer auf den digitalen Plattformen zunächst registrieren müssen, um sich für ein solches SCF-Programm qualifizieren zu können. Wird eine Rechnung durch den OEM bestätigt und für die frühzeitige Auszahlung freigegeben, so kann über die digitale Plattform nach einem vordefinierten Muster ein Skonto ,ausgehandelt" und die Rechnung nach Abzug des Skontos innerhalb der verhandelten Tage beglichen werden.

\section{Schlussbetrachtung}

Zusammenfassend lässt sich festhalten, dass das WCM in der kapitalintensiven und global vernetzten Automobilindustrie auch in Zukunft ein Dauerthema bleiben wird. Der globale, Automotive Supply Chain-bezogene Vergleich der WCM-Kennzahlen unterstreicht, dass die meisten Unternehmen in Bezug auf das Nettoumlaufvermögen noch viel Luft nach oben haben. Durch den Querschnittscharakter verlangt ein unternehmensübergreifendes WCM, dass OEMs und Zulieferer nicht nur die internen WCM-Zielkonflikte zwischen den einzelnen Unternehmensbereichen, sondern insbesondere auch jene mit den vor- und nachgelagerten Supply-Chain-Partnern (Kunden und Lieferanten) ausgleichen. Übereinstimmend mit der neoklassischen Theorie zeigt die Analyse der Automobilzulieferer beispielhaft auf, dass eine Reduktion des Working Capitals nur bis zu einem bestimmten Niveau einen positiven Effekt auf die Unternehmens-Performance ausübt. Für die in der Automobilindustrie tätigen Unternehmen besteht im WCM folglich eine zentrale Aufgabe darin, ihr Nettoumlaufvermögen mit den auf dem Markt verfügbaren SCF-Instrumenten gezielt auf das performancemaximierende Niveau hin zu steuern. Besonders für die WCM-Bezugspunkte Debitoren und Kreditoren existieren auf dem Markt bereits eine Vielzahl an technologisch ausgereiften SCF-Lösungen, auf die die Unternehmen zurückgreifen können (vgl. Hofmann/Wetzel, 2019). Dynamic Discounting als eine SCF-Lösungsoption beispielsweise unterstützt Unternehmen bei der Umsetzung einer unternehmensübergreifenden WCM-Strategie, indem Sie sich durch überdurchschnittliche Produkt- oder Service-Leistungen für ihre Supply Chain-Partner von anderen Mitbewerbern abheben können (z. B. durch die rasche Zahlung von Rechnungen). Je weiter sich SCF-Praktiken innerhalb der Automobilindustrie in Zukunft verbreiten, desto größer fällt auch das Optimierungspotenzial aus. In der Breite nutzen die Unternehmen der Automobilindustrie (insbesondere Tier 1 bis N Lieferanten) die am Markt angebotenen SCF-Lösungen immer noch verhältnismäßig zögerlich. Und dies, obwohl der Markt für SCF-Lösungen derzeit rasant
Implikationen für die Praxis

- Das Working Capital Management muss über die Supply Chain hinweg unter Einbezug der Lieferanten und Kunden optimiert werden, intra-organisationale Optimierungen sind nur bedingt effizient.

- Unternehmen mit überschüssiger Liquidität bzw. günstigen Finanzierungskosten sollten Supply Chain-Partner mit schlechteren Situationen bzw. Konditionen entlasten, indem sie ihr Zahlungs- resp. Rechnungsstellungsverhalten entsprechend anpassen.

- WCM Bumerang-Effekte entlang der Supply Chain erfordern eine ganzheitliche Betrachtung des Nettoumlaufvermögens.

- Unternehmen sollten Supply Chain Finance gezielt dazu nutzen, um ihr performancemaximierendes Niveau an Nettoumlaufvermögen zu erreichen.

wächst und innovative Alternativen zur Verfügung stehen. Von dieser Warte aus betrachtet sind Unternehmen dazu angehalten, unternehmensübergreifendes WCM nicht nur als operative Managementaufgabe zur Kostenreduktion zu verstehen, sondern als Mittel zum Zweck, um langfristige Unternehmensziele wie die Erhöhung des finanziellen Spielraums, die Optimierung der Liquidität, die Senkung von Finanzierungskosten sowie die Verbesserung der operativen Marge und Rentabilität entlang der Supply Chain zu erreichen. Schließlich befinden sich zukünftig nicht einzelne Unternehmen, sondern ganze Supply Chains im Wettbewerb zueinander.

\section{Infobox: Ablauf einer SCF-Optimierung}

1. Eigene Working Capital-Situation mithilfe eines Branchen-Benchmarkings analysieren.

2. Adäquates Working Capital-Niveau unter Berücksichtigung von Lieferanten und Kunden eruieren.

3. Geeignete Stoßrichtungen für eine SCF-Lösung evaluieren (inkl. Auswahl eines Lösungsanbieters).

4. Favorisierte SCF-Lösung mit wichtigen Lieferanten und Kunden erörtern.

5. Geeignete SCF-Maßnahmen anwenden und Lieferanten sowie Kunden „onboarden“.

6. Kontinuierliche Überwachung der Erfolgswirkung der SCF-Lösung.

7. Ggf. Nach-Justierung der SCF-Maßnahmen.

\section{Literatur}

- Hofmann, E./Wetzel, P., Working Capital Management Studie 2018-Supply Chain Finance Einführung, 5. Aufl., Zürich 2018.

- Hofmann, E./Wetzel, P., Working Capital Management Studie 2019-Supply Chain Finance Fortführung, 6. Aufl., Zürich 2019.

- Hofmann, E./Wetzel, P., Supply chain finance, financial constraints and corporate performance: An explorative network analysis and future research agenda, in: International Journal of Production Economics, 216. Jg. (2019), S. 364383. 


\section{Daten effizient nutzen.}

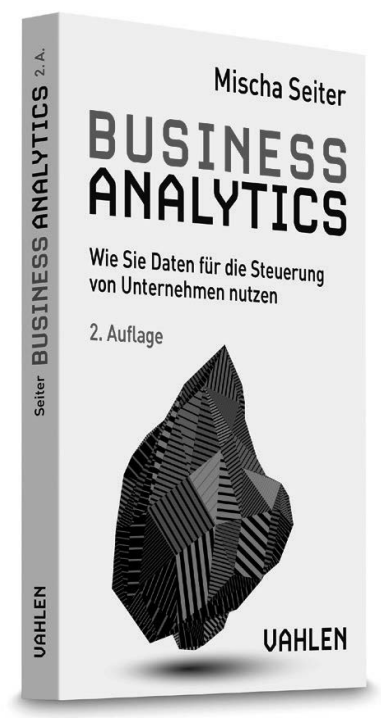

\section{Seiter}

Business Analytics

2. Auflage. 2019. VIII, 252 Seiten. Kartoniert $€ 49,80$ ISBN 978-3-8006-5871-8

\section{Dieses Buch}

vermittelt alle wesentlichen Aspekte von Business Analytics in kompakter Form. Den Rahmen dazu bilden vier Grundfragen:

1. Für welche betriebswirtschaftlichen Probleme sollen die knappen Analytics-Ressourcen eingesetzt werden?

2. Welche Ressourcen, also Daten, IT und Personal, sind zur Lösung der Probleme notwendig?

3. Mit welchen Algorithmen können die Probleme gelöst werden?

4. Wie müssen die Ergebnisse der Algorithmen interpretiert und visualisiert werden, damit Führungskräfte sie korrekt einsetzen können?

\section{9}

Für Führungskräfte, die ihr Verständnis von Datenanalyse erweitern und Analytics-Prozesse überblicken, planen und steuern wollen, ist das Buch ... ein Muss.

in getabstract zur Vorauflage

- Vazquez, X. H./Sartal, A./Lozano-Lozano, L. M., Watch the working capital of tier-two suppliers: a financial perspective of supply chain collaboration in the automotive industry, in: Supply Chain Management: An International Journal, 21. Jg. (2016), H. 3, S. 321-333.

- VDA, Automobilindustrie und Märkte, 2019, https://www.vda.de/de/themen/automobilindu strie-und-maerkte.html, Stand: 15.04.2019.

- Wetzel, P./Movcharenko, A., Net Working Capital: das adäquate Niveau, in: Controlling \& Management Review, 63. Jg. (2019), H. 6, S. 30-38.

\author{
Literaturtipps aus dem Online-Archiv \\ http://elibrary.vahlen.de \\ - Matthias Sure, Working Capital-Steuerung mit \\ integrierten Kennzahlen, Ausgabe 37/2015, \\ S. 7-12. \\ - Stephan Wagner und Pan Theo Grosse-Ruyken, \\ Working Capital Optimierung im Supply Chain \\ Management, Ausgabe 22/2010, S. 362-367.
}

\title{
Stichwörter
}

\# Dynamic Discounting \# Lieferantenfinanzierung \# Supply Chain Finance \# Unternehmensübergreifendes Working Capital Management

\section{Keywords}

\# Dynamic Discounting \# Holistic Working Capital Management \# Supplier Financing \# Supply Chain Finance

\section{Summary}

Supply chain finance (SCF) as an alternative form of financing extending the perspective of working capital management (WCM) to the entire supply chain. It offers companies within the automotive industry not only the prospect of an improvement in balance sheet figures and financing costs, but also a reduction in risks and increased transparency along the supply chain. Based on the example of the automotive industry, this article illustrates how a cross-company WCM, with the involvement of SCF, is becoming a success factor for the entire supply chain. 